\title{
EDITORIAL: LA FORMACIÓN DOCENTE
}

\section{Andrés Díaz Velasco}

La sección central del número 24 de la Revista Lúdica Pedagógica se ha dedicado a un tema que, aunque sumamente intrincado, es, en apariencia, de fácil aprehensión, sobre todo en el campo de la educación física, la recreación, el deporte y áreas afines, donde, por tradición, la formación ha implicado la existencia de un docente. Esto es, mientras que existen áreas donde el saber resulta más importante que el ser docente -ya que cualquier profesional con tales conocimientos puede así mismo enseñarlos- quien imparte una "clase" de educación física, recreación o deporte, en esencia brinda algo más que un saber. ¿Por qué?

Arriesgo dos respuestas: (i) porque el saber específico de estos campos está en la nebulosa, pasando (no solo históricamente) de la higiene y el aseo personal, al vigor moral a través del físico, a la regulación de los movimientos (conforme a prácticas específicas), al aprendizaje de normas que seguir, al desarrollo de gestos técnicos y a la compensación energética (donde caben el descanso, la diversión, el entretenimiento, etc.); y, (ii) porque estos campos no responden a una disciplina académica o científica de base, lo que además de hacer etéreo su saber enseñable, configura la situación paradójica de enseñar algo que no se sabe exactamente qué es, a la par de crear esto que se ha de enseñar en el maremágnum de posibilidades no disciplinarias. Generalmente, para un docente de estos campos es más fácil decir cuáles son sus objetivos o intenciones pedagógicas, que el saber que está, de algún modo, "transmitiendo". Lo cual es, sin duda, más una fortaleza que una debilidad.

De allí que, en estos campos, conceptos que han resultado clave en otros, tales como didáctica, saber docente e, incluso, lúdica, suenen a la vez tan naturales y tan ajenos. Y, por lo mismo, que la producción académica sobre la formación docente sea más bien indirecta, centrándose ora en temáticas como el juego, el cuerpo o la normatividad, ora en procesos como la organización curricular, la construcción de trabajos de grado y el establecimiento de énfasis de formación, como esta edición lo muestra. Lo que tal situación indica, es que el tema requiere la especialización investigativa y literaria, más, a la vez, el cuidado de no perder condiciones tan especiales como las que hemos señalado. El escenario académico de la educación física, el deporte, la recreación y afines, es tan rico y particular, que está servido como un banquete dionisiaco para el deleite de quien quiera ser parte.

Docente de tiempo completo, Licenciatura en Recreación, Universidad Pedagógica Nacional. Correo electrónico: andresdiazvelasco@gmail.com 
Así las cosas, cuestiones relacionadas con la diferencia entre el "maestro" y el "profesor público", o entre la "docencia" y la "enseñanza" o, incluso entre la "enseñanza" y la "instrucción" (o el adiestramiento); así como los problemas del saber "científico" de base, o el saber enseñable, o la construcción epistemológica de lo que sería posible enseñar (más allá, por ejemplo, de un gesto motor o de una actividad como tal), resultan fundamentales para profundizar en el significado de la formación docente dentro de los campos. El docente de educación física en la escuela (comúnmente uno de los preferidos por los estudiantes, si no el más, como suele indicarse) debe plantearse, o volver a hacerlo, una y otra vez, cuál es el sentido de su existencia (profesional, educativa, social, política...), para desde allí contribuir al replanteamiento constante del sentido de su formación. Y esto es lo que se ha propuesto la Revista Lúdica Pedagógica en este número.

Ahora bien, en la sección central de esta edición se encontrarán distintos puntos de vista que, por su diversidad, nos muestran el amplio espectro de lo posible. En primer lugar, y como es bien sabido, independientemente de que los hechos hayan sido unos y no otros, la historia es una versión particular de los mismos. Los métodos en la historiografía pretenden alcanzar este grado de precisión necesario para que la imaginación, la fantasía o la invención humana no modifiquen lo pasado bajo sus propios intereses o descuidos y, no obstante, siempre será privilegiado el relato de quien vivió, de algún modo, lo acontecido. Por ello, esta edición empieza con el documento del profesor Germán Preciado Mora, quien, como testigo, a través de fotografías, de su percepción y de otros estudiosos de la historia, narra su versión acerca de los inicios (alrededor de 1925) y el presente de la formación de docentes para la Educación física en Colombia; tarea que no es poca, pero que realiza de un modo sencillo y amable.

De acuerdo con lo que nos muestra el profesor Preciado, la Educación física en el país ha pasado por crisis tan extremas que la han hecho casi desaparecer, lo cual resulta paradójico dado los aportes que ha generado -según él- no solo en cuanto a la formación y especialización deportiva, sino a la formación de la niñez y de la juventud, a la salud y al bienestar de la sociedad colombiana, así como al incremento, cada vez mayor, de investigaciones y eventos que exponen su importancia para la formación de una sociedad mejor. La exposición del profesor nos muestra con cierto detalle las instituciones que actualmente se hacen cargo de la formación de docentes en Educación física, y la gran diversidad que poseen según características tanto contextuales, como epistemológicas y pedagógicas. Así, pues, pese a los deseos de homogeneización, y a la mirada peyorativa que aún parece existir en distintos medios académicos y gubernamentales, los diversos estudios y la formación docente en Educación física, en recreación y en deporte, están cada vez más fortalecidos y enraizados.

En seguida, producto de una investigación que reunió a dos grupos y a cuatro investigadores de la Facultad de Educación Física de la Universidad Pedagógica Nacional, el artículo La formación docente desde la normatividad, escrito por los docentes Sonia López Domínguez, Alfonso Martín Reyes, Juan Manuel Carreño Cardozo y Andrés Díaz Velasco, nos muestra un panorama actual del marco jurídico en que está envuelta la formación de docentes en educación física y en recreación. El artículo presenta de manera muy sucinta el cuerpo del proyecto de investigación realizado, muestra además la orientación tomada acerca del campo normativo, y finaliza con apreciaciones muy perspicaces sobre la situación en la que se encuentra la formación de docentes en Educación física (y áreas afines) en el país, sobre todo en relación con el sentido de la docencia y de la calidad. 
En tercer lugar, se presenta un artículo que se enlaza con los tres que le continúan, siguiendo la línea de la experiencia, de lo que se hace, en la formación de docentes en los campos de la Educación Física, la Recreación y el Deporte. El artículo denominado Reflexiones en torno a una práctica pedagógica en clave didáctica con y desde la motricidad en programas de formación de maestros, de los autores José Enver Ayala Zuluaga, Carlos Federico Ayala Zuluaga y Samuel de Souza Neto, da cuenta no solo de la importancia de cargar teórica y científicamente las prácticas pedagógicas, sino de orientarlas como verdaderos procesos de formación docente. Esto implica en sí una concepción de docente, además de claridad en lo que es su propósito dentro de la escuela. Asimismo, de este artículo, resulta fundamental la noción didáctica con y desde la motricidad, que obliga a hacerse una idea del sentido del cuerpo en movimiento.

Luego, en el artículo El trabajo fin de grado en la formación del maestro. La acción tutorial como pieza clave del proceso, de las autoras Magdalena Sáenz-deJubera-Ocón, Rosa Ana Alonso-Ruiz, María Ángeles Valdemoros-San-Emeterio, Eva Sanz-Arazuri y Ana Ponce-de-León-Elizondo, se presenta una interesante propuesta que se viene realizando en la Universidad de La Rioja (España). Tal propuesta ha adoptado como plataforma el "trabajo fin de grado", un escenario de formación innovador (para el contexto español) en el que se da la posibilidad a un tutor de orientar a los estudiantes en la conformación y desarrollo de propuestas de intervención didáctica, las cuales, en este caso, giran en torno a cuatro grandes ejes: ocio cultural, juegos tradicionales, actividad física y lectura compartida, conforme al proyecto de ocio saludable en familia.

Para completar esta tanda de artículos relacionados con experiencias en formación de docentes, el texto Creando puentes entre la formación universitaria y la no universitaria a través de la recreación en un grupo de trabajo, de María Beatriz Páramo-Iglesias, Manuela Raposo-Rivas y Esther Martínez-Figueira, describe una interesantísima experiencia en la que se aboga por una transformación profunda de la docencia y, por tanto, de la formación de docentes. Según dicen las autoras, "el trabajo conjunto entre la escuela, la universidad y el centro de formación ofrece la posibilidad de reflexionar sobre la competencia de aprender a aprender en busca de una base para posteriores aprendizajes, y, específicamente, en educación infantil en relación con la autonomía y el cuerpo, base para el conocimiento". Vale la pena resaltar el sentido de la recreación como metodología para aprender a aprender, concibiéndola "como un conjunto de dinámicas y juegos manipulativos, de pensamiento, razonamiento, role playing, etc., que permita que los diferentes agentes expresen sus sentimientos, su parecer, sus idearios y sus frustraciones sobre una temática concreta, con el fin de buscarle solución a esa problemática".

Terminamos la sección central de este número con dos artículos que tratan, en la formación docente, dos temas ineludibles y tradicionalmente de altísima relevancia para los campos de la educación física, la recreación, el deporte y afines. En el primero, Concepciones sobre cuerpo en la formación de maestros de Educación física de la Universidad de los Llanos, el profesor Fernando Campo Polo nos muestra una situación que, aunque se refiere a un caso particular, es fácilmente aplicable a otros contextos de formación de la educación física, ya que aborda los conceptos, tradicionales y novedosos, en los que se sustenta un currículo. La importancia 
del cuerpo entre estos conceptos, como es natural, varía según el tiempo, siendo, no obstante, una línea de análisis decisiva en las prácticas discursivas de la formación de maestros en la educación física, por lo que el trabajo del profesor Campo resulta esencial para comprender la transformación del campo según su concepción.

El último de los artículos, El juego en el ámbito disciplinar de la educación física y la formación de sus docentes, del profesor José Orlando Pachón, analiza el sentido del juego y su importancia en la formación de licenciados en Educación física, a partir de la diferencia entre educar para el juego y educar a través del juego. La diferencia de estas nociones da cuenta de una distinción conceptual más profunda -entre el juego como herramienta o actividad y el juego como un valor de la existencia humana-, la cual demarca tanto una orientación curricular de formación de docentes, como el horizonte de trabajo de estos. El tema no es menor en cuanto a la historia de la educación física se refiere, ni mucho menos en relación con la importancia del juego para el hombre en sí, pero el modo en el que el profesor Pachón trata el tema permite que nos mantengamos en el plano de lo concreto, esto es, de los planteamientos teóricos más influyentes, de los currículos influenciados y de los influenciables docentes en formación.

Como complemento a esta sección central, en la sección institucional se presenta el artículo La recreación en la práctica pedagógica I y II, de las profesoras Janeth Castañeda y Viviana Sierra, que expone algunos de los desarrollos de las prácticas pedagógicas en la Licenciatura en Recreación y Turismo de la Universidad Pedagógica Nacional, a partir del trabajo realizado por algunos estudiantes en un colegio en particular y conforme al contexto de la formación de docentes en la Licenciatura. Es interesante analizar en esta perspectiva, las denominadas "herramientas recreativas", así como la idea de tener un "perfil" de formador y la importancia superlativa del lugar de práctica.

De este modo, el número 24 de la Revista Lúdica Pedagógica, al adentrarse en el tema de la formación de docentes para los campos en cuestión, invita a sus lectores a continuar con el estudio dedicado y riguroso que se requiere, siempre que lo que nos parece más normal, sea puesto en duda y alterado constantemente en pro de la innovación. El número incluye tres artículos más que invito, igualmente, a leer con detenimiento, esperando que todo este complazca a los lectores en su deseo infinito de saber. 